\title{
Geburtshilflich-gynäkologische Gesellschaft in Wien
}

\section{Sítzung vom 13. März 1934.}

1. G. Halter: a) Adenokarzinom und Schwang •erschaft. Bericht über eine 32jährige Patientin. Anamnese o. B. 1930 an der Klinik (weiland Prof. Peham) operiert. Entfernung der linken Adnexe wegen Kystoma multiloculare. März 1933: Ausbleiben der Menstruation, Schmerzen im Unterbauch. Harndrang. Diagnose: Gravidität -f r. Adnextumor. Die Operation ergibt eine Gravidität von zwei Monaten plus r. zystischen Tumor, der an der Innenwand papilläre Exkreszenzen aufweist. 14. November 1933 spontan entbunden. Histologische Untersuchung ergibt maligne Degeneration. In der Literatur finden sich 52 Fälle von malignen Ovarialtumoren während der Schwangerschaft. Auf der Klinik 2 Fälle seit 20 Jahren. In diesem Falle scheint die Schwangerschaft das Tumorwachstum gefördert zu haben. Beachtenswert ware der Fall noch deshalb, weil es, obwohl durch Operation das corpus luteum gravidit. entfernt wurde, zu keinem Abort gekommen ist. L. M. war am 8. Februar 1933. Am 14. April wurde das corpus luteum durch Operation entfernt, am 17. April traten krampf-artige Schmerzen mit leichter Blutung auf. Es wurde Luteogan injiziert und weiterhin Luteogantabletten dreimal täglich gegeben, im ganzen 120 Tabletten. Es dürfte auch clieser Therapie der Erfolg zuzuschreiben sein.

Aussprache.

Weibel berichtet von einem Fall, den er vor wenigen Wochen zu beob-achten Gelegenheit hatte. 40jährige Nullipara, die sich ein Kind gewünscht hatte und deshalb ständig unter Beobachtung stand. Patientin wurde gravid und bis zum 5. Monat regelmäßig digital untersucht, wobei stets ein normaler Tastbefund erhoben wurde. Am 7. Monat der Gravidität traten Schwellungen der Beine auf. Abdomen größer als es der Zeit entsprochen hätte. R. R. normal, kein Albumen. Patientin verfiel zusehends, so daß vom Vortragenden eine Sectio caesarea abgelehnt wurde, da befürchtet werden mußte, das Patientin am Tiseh bleibe. Da sich der Zustand zusehends verschlechterte, wurde doch zur Operation geraten, doch traten am Tage, bevor die Operation hätte ausgeführt werden sollen, Wehen auf. Nach 7 Stunden Spontangeburt. Die bimanuelle Untersuchung ergab nun beiderseits mannsfaustgroße Ovarialtumoren, gleichzeitig zahlreiche Knoten im ganzen Abdomen, Aszites. Punktion 61 Flüssigkeit. Patientin ist 2 Wochen nach dem Partus gestorben. Das rasche Wachstum des Tumors dürfte im 3.-4. Schwanger-schaftsmonat entstanden sein, ist mit der Schwangerschaft in Zusammen-hang zu bringen. Hätte man früher operiert, hätte man den Uterus ent-f ernen müssen, da sonst die Patientin an einer Sepsis zugrunde gegangen ware.

Joachimoviís zeigt das histologische Präparat eines kleinen Kystoma pappilifer., das anläßlich der Operation einer Gravidit. extrauterin. gefunden wurde. Gebui'tshilflich-gynäkologische Gesellschaft in Wien. 177

b) Expulsion des Corpus luteum. Halter zeigt das Präparat von beider-seitig entzündlich veränderten Adnexe, die bei einer Frau, die wegen chronischer Appendizitis und Retroflexio uteri fix. zur Operation kam, auch entfernt wurden; es fand sich darin ein ausgestoßenes corpus luteum. Darstellung einer schematischen Zeichnung. Genese der Expulsion. Drei Vorbedingungen müssen gegeben sein: Abnorme Größe, loekere Verbindung mit dem 
Ovarialbett, mechanische Momente. Untersuchung durch den Arzt oder entzündliche Prozesse führen dann zu Expulsion.

2. R. Schweigí: Torsion eines Myoms mit Aehsendrehung des Uterus.

52jährige Patientin; seit 15 Jahren Myom, seit Herbst 1933 Schmerzen.

Pulslose Patientin mit Zeiohen der Dilatation des Herzens. Tumor bis

processus x. Diagnose: Uterus myomatosus. Nekrose ? Aohsendrehung?

Operation 20. Dezember. Nach Beseitigung der kardialen Insuffizienz

supravaginale Amputation. Torsion des corpus uteri um 540 . Uterus

faustgroß, übergehend in einen gestielten $3 \frac{1}{2} \mathrm{~kg}$ schweren Tumor. De

monstration des Präparates.

Aussprache. Keller: Beim Rinde kommen Torsionen häufig im Eröffnungsstadium vor. Die Mutterbänder winden sich um die Cervix uteri. Es kann sogar die Gebärmutter dadurch amputiert werden. Die Tiere werden durch Zurückdrehen manchmal gerettet. Ohne Therapie oft Exitus. 1 Fall von Dermoid beim Rinde mit sechsmaliger Drehung des einen Gebärmutter-horns. Er zeigt verschiedene Bilder.

P. Singer (a. G.): Ein Fall von Gíraviditas infundibularis. 13 Fälle von Graviditas infundibularis finden sich im Handbuch von Halban-Seitz, aber darunter nur einer mit Sitz des Eies in der fimbria ovarica. Seit 1926 sind noch einige Fälle dazugekommen. Hier: 19jährige Patientin, 1. Periode im 15. Jahre, 3 Wochen, 4 Tage. Kein Partus, kein Abortus. Uterus in Retroversion. Heißluft. Stuhlregelung. Patientin wurde in der Ambulanz ohnmächtig als Zeichen einer schweren innerenBlutung, Douglas vorgewölbt. Operation: 1 1/8-2 1 Blut im Abdomen; im rechten Ovarium corpus luteum. Teile der linken Tube mit einer Umstechung abgetragen. Im Infundibulum ein haselnußgroßer glatter Tumor, histologisch: Tubarabort. Es dürfte sich um eine äußere Eiüberwanderung gehandelt haben.

W. Schiller: Zur Histogenese der Brenner-Tumoren. Ausführliche Besprechung in Anlehnung an Robert Meyer. Ausführlicher Bericht über die Fälle der Klinik Professor Weibel mit sehr interessanten Bildern. (Er-scheint ausführlich.)

O. Reichelt: Röntgendiagnose der Anencephalie bei Hydramnion. Hydramnion bei Patientin, die seit zwei Tagen keine Kmdesbewegungen spurt. Kolpeurynther eingelegt; wird bald ausgestoßen. Röntgen zeigt die Mißbüdung; bei Blasensprung viel Fruchtwasser, Geburt in Gesichtslage, Frucht zeigt Anencephalie und Rachischisis. Röntgenbilder.

Aussprache.

Heidler und Weibel berichten über ähnliche Fälle. Heidler und Reichelt können sich über die Berechtigung des Kolpeurynthers in diesem Falle nicht einigen. Hofstätter-Wien.

Monatsschrift f, Geburtshülfe u. Gynäkologie. Bd. ХСVГI. Heft 3 (Jani 1934). 